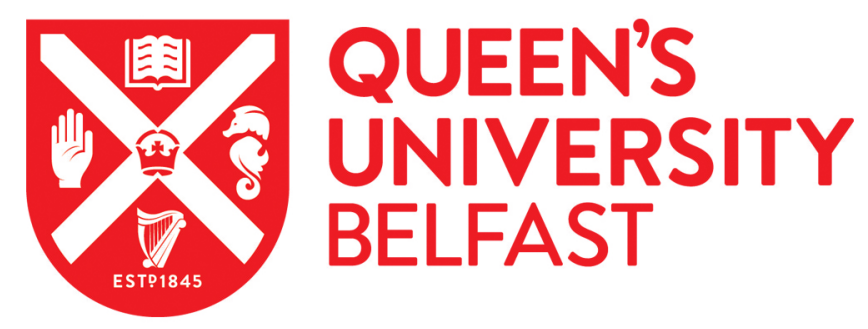

\title{
CHARMM-GUI Membrane Builder for Complex Biological Membrane Simulations with Glycolipids and Lipoglycans
}

\author{
Lee, J., Patel, D. S., Ståhle, J., Park, S-J., Kern, N. R., Kim, S., Lee, J., Valvano, M. A., Cheng, X., Holst, O., \\ Knirel, Y. A., Qi, Y., Jo, S., Klauda, J. B., Widmalm, G., \& Im, W. (2018). CHARMM-GUI Membrane Builder for \\ Complex Biological Membrane Simulations with Glycolipids and Lipoglycans. Journal of chemical theory and \\ computation, 15(1), 775-786. https://doi.org/10.1021/acs.jctc.8b01066
}

Published in:

Journal of chemical theory and computation

Document Version:

Peer reviewed version

Queen's University Belfast - Research Portal:

Link to publication record in Queen's University Belfast Research Portal

Publisher rights

Copyright 2018 ACS. This work is made available online in accordance with the publisher's policies. Please refer to any applicable terms of use of the publisher.

\section{General rights}

Copyright for the publications made accessible via the Queen's University Belfast Research Portal is retained by the author(s) and / or other copyright owners and it is a condition of accessing these publications that users recognise and abide by the legal requirements associated with these rights.

Take down policy

The Research Portal is Queen's institutional repository that provides access to Queen's research output. Every effort has been made to ensure that content in the Research Portal does not infringe any person's rights, or applicable UK laws. If you discover content in the Research Portal that you believe breaches copyright or violates any law, please contact openaccess@qub.ac.uk. 


\section{CHARMM-GUI Membrane Builder for Complex Biological Membrane Simulations with Glycolipids and Lipoglycans}

Jumin Lee ${ }^{1}$, Dhilon S. Patel ${ }^{1}$, Jonas Ståhle ${ }^{2}$, Sang-Jun Park ${ }^{1}$, Nathan R. Kern ${ }^{1}$, Seonghoon $\mathrm{Kim}^{1}$, Joonseong Lee ${ }^{1}$, Xi Cheng ${ }^{3}$, Miguel A. Valvano ${ }^{4}$, Otto Holst ${ }^{5}$, Yuriy A. Knirel ${ }^{6}$, Yifei Qi ${ }^{7}$, Sunhwan $\mathrm{Jo}^{8}$, Jeffery B. Klauda ${ }^{9}$, Göran Widmalm², and Wonpil $\mathrm{Im}^{1^{*}}$

${ }^{1}$ Departments of Biological Sciences and Bioengineering, Lehigh University, Bethlehem, PA 18015, USA

${ }^{2}$ Department of Organic Chemistry, Arrhenius Laboratory, Stockholm University, SE-106 91 Stockholm, Sweden

${ }^{3}$ State Key Laboratory of Drug Research, Shanghai Institute of Materia Medica, Chinese Academy of Sciences, 555 Zuchongzhi Road, Shanghai 201203, China

${ }^{4}$ Wellcome-Wolfson Institute for Experimental Medicine, Queen's University Belfast, BT9 7BL, United Kingdom.

${ }^{5}$ Division of Structural Biochemistry, Research Center Borstel, Airway Research Center North, Member of the German Center for Lung Research (DZL), D-23845 Borstel, Germany

${ }^{6} \mathrm{~N}$. D. Zelinsky Institute of Organic Chemistry, Russian Academy of Sciences, 119991 Moscow, Russia

${ }^{7}$ Shanghai Engineering Research Center of Molecular Therapeutics and New Drug Development, School of Chemistry and Molecular Engineering, East China Normal University, Shanghai, 200062, China

${ }^{8}$ Leadership Computing Facility, Argonne National Laboratory, Argonne, IL 60439, USA

${ }^{9}$ Department of Chemical and Biomolecular Engineering and the Biophysics Graduate Program, University of Maryland, College Park, MD 20742, USA

*To whom correspondence should be addressed: Tel: +1-610-758-4524; Fax: +1-610-758-4004; e-mail: wonpil@lehigh.edu

Keywords: molecular dynamics, lipopolysaccharides, glycosphingolipids, bacterial outer membranes 


\begin{abstract}
Glycolipids (such as glycoglycerolipids, glycosphingolipids, and glycosylphosphatidylinositol) and lipoglycans (such as lipopolysaccharides (LPS), lipooligosaccharides (LOS), mycobacterial lipoarabinomannan, and mycoplasma lipoglycans) are typically found on the surface of cell membranes and play crucial roles in various cellular functions. Characterizing their structure and dynamics at the molecular level is essential to understand their biological roles, but systematic generation of glycolipid and lipoglycan structures is challenging because of great variations in lipid structures and glycan sequences (i.e., carbohydrate types and their linkages). To facilitate the generation of all-atom glycolipid/LPS/LOS structures, we have developed Glycolipid Modeler and LPS Modeler in CHARMM-GUI (http://www.charmm-gui.org), a webbased interface that simplifies building of complex biological simulation systems. In addition, we have incorporated these modules into Membrane Builder, so that users can readily build a complex symmetric or asymmetric biological membrane system with various glycolipids and LPS/LOS. These tools are expected to be useful in innovative and novel glycolipid/LPS/LOS modeling and simulation research by easing tedious and intricate steps in modeling complex biological systems, and shall provide insight into structures, dynamics, and underlying mechanisms of complex glycolipid-/LPS-/LOS-containing biological membrane systems.
\end{abstract}




\section{INTRODUCTION}

Cell membranes have a wide variety of lipids that provide a matrix to host integral membrane proteins and recruit peripheral membrane proteins, thus actively participating in cellular membrane functions together with these proteins ${ }^{1,2}$. The complexity of biological membranes arises from the considerable heterogeneity in the spatial distribution of lipids and proteins in the cell membrane and between the bilayer leaflets. Some of the most complex chemical structures of lipids contain sugars, known as glycolipids or lipoglycans. For example, many eukaryotic membranes contain an outer leaflet rich in amphiphilic glycolipids, which are involved in critical biological processes, such as signal transduction, cell-cell recognition, adhesion, and cell death ${ }^{3-6}$. Gram-negative bacteria contain either lipopolysaccharides (LPS) or their shorter relatives, lipooligosaccharides (LOS), which provide highly complex and diverse structures in the outer leaflet of the outer membrane (OM), whereas the inner leaflet contains a mixture of phospholipids, such as phosphatidylethanolamine, phosphatidylglycerol, and cardiolipin?

Glycolipids can be subdivided into several subclasses: (i) glycosphingolipids (GSLs), (ii) glycoglycerolipids (GGLs), and (iii) glycosylphosphatidylinositol (GPI). LPS and LOS are by definition lipoglycans rather than glycolipids. GSLs, in which the lipid part is a ceramide (Cer), are abundant in the plasma membrane of cells in higher eukaryotes and often cluster to form 'lipid rafts' together with specific proteins (e.g., GPI-anchored proteins and proteins involved in signal transduction). GSLs are particularly abundant in nerve cells, playing important roles in rapid nerve conduction. GGLs utilize a glycerol to anchor the glycan to the cell membrane. GPI contains a complex oligosaccharide and anchors proteins to membranes. GPIs are found in protozoa, fungi, and humans, and their loss may lead to hemolytic anemia in humans. LPS protects Gram-negative bacteria from host innate immune defenses and provide structural stability to the $\mathrm{OM}$ via ionic interactions with divalent metal cations. In addition, the O-antigen polysaccharides that form the outermost region of the LPS molecules in many bacteria can contribute to protection against host defenses and bacteria-bacteria interactions in biofilms. Further, some repetitive oligosaccharides of the O-antigen and the outer core of LOS mimic host-like antigen structures, such as blood-group determinants or sialic acid-containing epitopes, help bacteria evade immune mechanisms. For example, Campylobacter jejuni (C. jejuni) mimics ganglioside GSLs in its outer core, which can lead to autoimmune diseases, such as the Guillain-Barré syndrome ${ }^{9,10}$. The lipid A part of LPS/LOS, which anchors these molecules to the OM, is toxic to higher animals including humans, depending on its structure, by eliciting potent inflammatory responses.

Despite their importance, generating glycolipid/lipoglycan structures for molecular modeling is challenging because of complex glycan sequence variations and diverse lipid types. Moreover, preparing molecular simulation systems containing glycolipids/lipoglycans is more difficult, as it requires appropriate force fields (FFs) for all necessary carbohydrates, lipid types, and chemical modifications. Consequently, there are much fewer simulation studies using glycolipids/lipoglycans compared to those with phospholipids. Previous glycolipid computational studies used structures based on those available from the PDB ${ }^{11,12}$, coarse-grained (CG) models ${ }^{13,14}$, or model structures generated via complicated ways ${ }^{15-18}$. However, the glycolipid/lipoglycan types available in the PDB or CG models are limited, and a customized building procedure is often arduous to follow. Several web-based tools, such as SWEET II $^{19}$, 
GLYCAM $^{20}$, and Glycan Modeler (http://glycanstructure.org), as well as stand-alone programs, such as POLYS ${ }^{21}$, CarbBuilder ${ }^{22}$, and doGlycans ${ }^{23}$ have been developed to facilitate glycan structure modeling. However, most of them, except SWEET II and doGlycans, do not support modeling of glycolipid moieties. Even SWEET II, which supports various lipid types including diacylglycerol (DAG), alkylacylglycerol, Cer, and acyl chains for lipid A, can only accept text input and do not offer diverse lipid tail variations. Further, significant post-processing efforts are required to use the generated structures for molecular dynamics (MD) simulation because SWEET II does not provide corresponding FF parameters. doGlycans provides structure and topology files for glycolipids, glycoprotein, and polysaccharides. However, for glycolipids, doGlycans requires users to prepare the structure, topology, and parameters of the lipid. In addition, doGlycans provides only molecular topology, so that any force field parameters for glycan-lipid linkages should exist in any desired force field to use generated glycolipids. As all the aforementioned tools are for modeling single glycan/glycolipid/lipoglycan structures, building a simulation system with the generated glycolipid/lipolgycan structures requires additional molecular packing tools, such as PACKMOL ${ }^{24}$ or MembraneEditor ${ }^{25}$. Therefore, despite that a glycolipid-/lipoglycan-containing membrane system could be prepared by a combination of these tools, preparing a complete FF parameter set for the system is labor-intensive and errorprone. The CG modeling tool insane ${ }^{26}$ provides a CG membrane model with glycolipids and phospholipids. However, insane supports only six glycolipid types (with various lipid tail lengths) at the time of writing this paper, and the conversion from a CG glycolipid model to an all-atom model is not straightforward.

Since its first release in 2007, CHARMM-GUI Membrane Builder ${ }^{27-30}$, a publicly available web-based tool (http://www.charmm-gui.org/input/membrane), has greatly facilitated the generation of complex membrane systems. Its first implementation allowed users to build a protein-membrane complex system with three lipid types ${ }^{28}$. After continuous development and update, Membrane Builder now supports heterogeneous bilayers, with or without proteins, using more than 400 lipid types including phospholipids, phosphoinositides, cardiolipin, sphingolipids, bacterial lipids, ergosterol, fatty acids, and detergents, which allows users to build biologically realistic and experimentally comparable membrane systems. Importantly, Membrane Builder also provides well-validated simulation inputs for various MD programs ${ }^{31}$, such as $\mathrm{CHARMM}^{32}$, NAMD $^{33}$, GROMACS ${ }^{34}$, AMBER ${ }^{35}$, GENESIS ${ }^{36}$, LAMMPS $^{37}$, Desmond ${ }^{38}$, OpenMM ${ }^{39}$, and CHARMM/OpenMM ${ }^{40}$, allowing users to perform MD simulation with their familiar tool. In this work, we describe the methods developed to build complex glycolipids (Glycolipid Modeler, http://www.charmm-gui.org/input/glycolipid, and LPS Modeler, http://www.charmmgui.org/input/lps) and to incorporate them into the Membrane Builder workflow for building a membrane system with any glycolipid/LPS/LOS type(s). Combined with Glycan Reader ${ }^{41,42}$ in CHARMM-GUI, which allows modeling of glycoproteins and carbohydrate ligands in a PDB file, Membrane Builder provides the most comprehensive tool for glycoconjugate modeling and simulation in a membrane environment. To illustrate the power of the updated Membrane Builder for various types of glycolipid-/LPS-/LOS-containing membrane simulations, we have built and simulated (i) GPI-anchored CD59 in human plasma membranes ${ }^{43}$, (ii), axonal membranes in human neuron cells with various gangliosides ${ }^{44,45}$, (iii) C. jejuni $\mathrm{OM}^{46,47}$, and (iv) BtuB in Escherichia coli (E. coli) OM models ${ }^{48}$, in which systems (ii) and (iii) are associated with the Guillain-Barré and Miller-Fisher syndromes. 


\section{METHODS}

Glycolipid Modeler. Glycolipid Modeler adopts a graphical user interface (GUI), allowing users to easily check and modify the glycolipid sequence (i.e., lipid type, carbohydrate types, and their linkages). The input is a glycolipid sequence selected from the pre-defined glycolipid sequences or manually specified by the user in the "Glycan Sequence" panel (Figure 1 A). Currently, there are 184 pre-defined glycolipids whose glycan sequences are experimentally identified $^{49-52}$, and each pre-defined glycolipid is categorized into 11 groups based on its glycan sequence and lipid type (Table 1). According to the lipid type, glycolipids are classified into GGLs, GSLs, and $\mathrm{GPI}^{8}$. While GGLs have DAG as the lipid moiety, GSLs are based on Cer, and GPI can have either $\mathrm{PI}-\mathrm{DAG}$ or $\mathrm{PI}-\mathrm{CER}^{50}$. Although there are only a few types of GGLs in nature, they are particularly important, as GGLs are the major glycolipids in plants and bacteria ${ }^{53}$. In contrast, GSLs are in vertebrate tissues. GPIs are in various eukaryotic cells and especially abundant in protozoa ${ }^{50}$. While GPIs alone can act as surface antigens or receptors, a GPI mostly exists as a linker to the C-terminus of a protein via ethanolamine phosphate ${ }^{54,55}$. These GPI-linked proteins play important biological roles as enzymes, adhesion molecules, regulatory proteins, receptors, and prion proteins ${ }^{50,56}$. Glycolipid Modeler provides a canonical GPI form and users can modify it if necessary. Linking GPI to proteins is possible in the PDB manipulation step in CHARMMGUI and separately described below.

In SphinGOMAP ${ }^{51}$, there are more than 200 GSL types reported. Based on the sugar type attached to Cer or the substitutions on a sugar, GSLs are classified into multiple subcategories (Figure S1). Galactocerebroside (GalCer) has a galactose (Gal) in a $\beta$ configured linkage to Cer, and a glucocerebroside (GlcCer) has a glucose (Glc) in a $\beta$-anomeric linkage to Cer. GlcCer can be further categorized by addition of Gal, forming a lactosylceramide (LacCer). LacCer is a branch-point for the core glycan sequence of vertebrate GSL families (ganglio-, globo-, isoglobo-, lacto-, and neolacto-series).

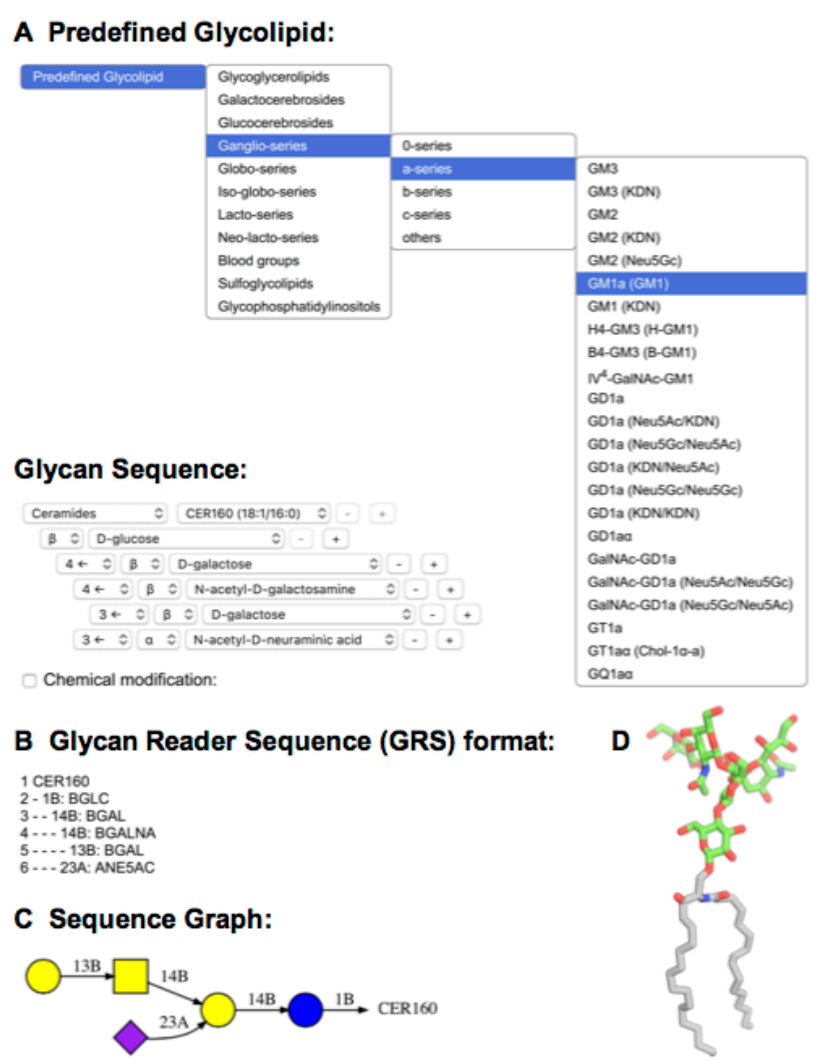

Figure 1. Illustrative snapshots of Glycolipid Modeler. (A) Users can build a glycolipid sequence by selecting one of e pre-defined glycolipids or by specifying their own sequence. In this illustration, GM1 is selected from the predefined glycolipids. The selected glycolipid sequence is also displayed using (B) Glycan Reader Sequence (GRS) format and (C) the symbolic representation. (D) The output structure of GM1. The lipid moiety is represented in gray, and the oligosaccharides are shown in green. 
Table 1. Pre-defined glycolipid classification in Glycolipid Modeler.

\begin{tabular}{|c|c|c|c|}
\hline Groups & Subgroups & \# glycolipids $^{\dagger}$ & Examples \\
\hline Glycoglycerolipids & - & 3 & MGDG, DGDG, SQDG \\
\hline Galactocerebrosides & - & 3 & GalCer, Sulfatide, $\|^{3}-\mathrm{SO}_{4}$-di-GalCer \\
\hline Glucocerebrosides & - & 2 & GlcCer, LacCer \\
\hline \multirow[t]{5}{*}{ Ganglio-series } & 0 -series & 15 & GA2, GM2 $\alpha$, GA1, GM1 $\alpha$, GM1b, GD1c, $\ldots$ \\
\hline & a-series & 23 & GM3, GM2, GM1, GD1a, GD1a $\alpha$, GT1a, ... \\
\hline & b-series & 16 & GD3, GD2, GD1b, GT1b, Chol-1 $\beta$, GT1b $\alpha, \ldots$ \\
\hline & c-series & 8 & GT3, GT2, GT1c, GQ1c, GP1c, GP1c $\alpha, \ldots$ \\
\hline & others & 1 & GM4 \\
\hline Globo-series & - & 17 & Gb3, Gb4, Forssman, SSEA-3, SSEA-4, ... \\
\hline Isoglobo-series & - & 3 & iGb3, iGb4, IV3-SO $-\mathrm{SO}_{4}$-iGb4 \\
\hline Lacto-series & - & 27 & Lc3, LcGg4, Lc4, A1-LacCer, Lea-LacCer, ... \\
\hline Neolacto-series & - & 54 & nLc4, nLc5, nLc6, A2-LacCer, Le ${ }^{\mathrm{x}}$-LacCer, ... \\
\hline \multirow{3}{*}{ Blood groups } & ABO & 23 & A1-LacCer, B1-LacCer, H1-LacCer, A1-Lc4, ... \\
\hline & Lewis & 35 & 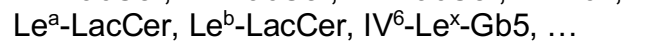 \\
\hline & $\mathrm{P}$ & 4 & $\mathrm{P}^{\mathrm{k}}$ antigen, $\mathrm{P}$ antigen, $\mathrm{P} 1$ antigen, $\mathrm{PX} 2$ antigen \\
\hline Sulfoglycolipids & - & 10 & Sulfatide, $\mathrm{IV}^{3}-\mathrm{SO}_{4}-\mathrm{Gb} 4, \|^{3}-\mathrm{SO}_{4}-\mathrm{GA} 1, \ldots$ \\
\hline Glycophosphatidylinositols & - & 12 & GPI-core, $T$. brucei VSG, Human PrP, ... \\
\hline Total & & $184^{\ddagger}$ & \\
\hline
\end{tabular}

tSome glycolipids are categorized into the multiple groups.

\$The total number of unique glycolipids.

Most glycolipids fall into one of the aforementioned groups, but some can be further categorized when they contain a chemical modification or certain glycan motifs. For example, some antigens expressed on the surface of human red blood cells contain specific glycan motifs, the so-called blood group antigens. There are 36 blood group systems discovered to date ${ }^{57}$. Among them, ABO, P, and Lewis blood group systems (Figure S2) include glycolipid-type antigens, and Glycolipid Modeler provides 62 blood group antigens in the pre-defined glycolipids (Table 1). Sulfoglycolipids are glycolipids with at least one sulfate group attached to any carbohydrate site. The sulfate group in sulfoglycolipids provides additional negative charges on the cell surface, contributing to molecular interactions during cell recognition and membrane microdomain formation ${ }^{58}$. There are 10 pre-defined sulfoglyolipids including the sulfatide, which is one of the most important lipid components in the nervous system ${ }^{59}$.

Glycolipid Modeler uses the common literature name for each pre-defined glycolipid whenever possible. For instance, a glycolipid having a sequence of $\operatorname{Gal}(\beta 1-3) \operatorname{GalNAc}(\beta 1$ $4)[N e u 5 A c(\alpha 2-3)]$ Gal $(\beta 1-4)$ GlcCer (GalNAc for $N$-acetyl-D-galactose and Neu5Ac for $N$-acetylneuraminic acid) is commonly referred to as GM1. However, many of pre-defined glycolipids do not have a common literature name. In such cases, Glycolipid Modeler provides a name as a derivative of a structurally closest glycolipid having a common name. ${ }^{8}$ For example, a glycolipid having a sequence of $\operatorname{GalNAc}(\beta 1-4) \operatorname{Gal}(\beta 1-3) \operatorname{GaINAc}(\beta 1-4)[\mathrm{Neu} 5 \mathrm{Ac}(\alpha 2-3)] \mathrm{Gal}(\beta 1-4) \mathrm{GlcCer}$ shares its sequence with GM1 up to the fourth sugar (count starts from the glycan attached to Cer), and GalNAc is attached to the fourth sugar with a $\beta$ - $(1 \rightarrow 4)$-linkage. Therefore, it is named IV $\mathrm{V}^{4}$-GalNAc-GM1, where IV and a superscript 4 are the position and glycosidic linkage site of the sugar to which GalNAc is attached, respectively.

The sequences of the pre-defined glycolipids are stored in Glycan Reader Sequence (GRS) format ${ }^{42}$ (Figure $1 \mathrm{~B}$ ). When a user selects a pre-defined glycolipid, the corresponding glycolipid sequence is sent to the client side to display the sequence (under "Glycan Sequence" in Figure $1 \mathrm{~A}$ ), the GRS format (Figure $1 \mathrm{~B}$ ), and its symbolic representation (Figure $1 \mathrm{C}$; based on glycan symbol nomenclature ${ }^{60}$ ). Figure 1 illustrates how one can generate a 
ganglioside GM1 structure (Figure 1 D) by simply clicking "GM1a (GM1)" in the pre-defined glycolipid list. A tutorial page illustrating how to generate a GM1 glycolipid structure is available at http://www.charmm-gui.org/tutorial/glycolipid.

If a glycolipid sequence of user's interest is not found in the pre-defined list, a custom sequence can be supplied either by modifying the existing sequences or supplying a new sequence under the "Glycan Sequence" panel. The panel provides options to add, remove, or modify sugar types, glycosidic linkages, and chemical modifications (such as sulfation and $O$ acetylation) at specific carbohydrate sites. In addition, Glycolipid Modeler supports various lipid types and allows users to change lipid types for a selected glycolipid. Currently, there are 51 lipid types with various tail lengths, 13 carbohydrate types, and 7 common chemical modifications available (Table S1). Note that both GRS format and symbolic representation are updated as the glycolipid sequence is changed, so one can easily verify the updated glycolipid sequence.

For a given glycolipid sequence, Glycolipid Modeler generates an all-atom structure using $\mathrm{CHARMM}^{32}$ based on common glycosidic torsion angles stored in the internal coordinate (IC) information in the CHARMM36 FF for lipids ${ }^{61,62}$ and carbohydrates ${ }^{63}$. The CHARMM input is automatically created based on the glycolipid displayed under the "Glycan Sequence" panel; see Figure S3 for the CHARMM input for GM1 in Figure 1. The CHARMM input, the glycolipid structure, as well as the topology and parameter files (for CHARMM, GROMACS, and AMBER) are downloadable in a single tar file ("download.tgz"). These files can be used for a MD simulation study of a glycolipid-containing system using various simulation packages.

Linking GPI to proteins. As mentioned in the previous section, a GPI moiety is usually linked to the C-terminus of a protein, anchoring the protein on the membrane surface. Since Glycolipid Modeler can only handle the generation of a glycolipid structure, another tool is necessary to manage both GPI and a protein and link them together. PDB Reader \& Manipulator ${ }^{27,64}$, http://www.charmm-gui.org/input/pdbreader, is the first CHARMM-GUI module that allows reading and manipulating a PDB biomolecular structure in a user-friendly web interface. PDB Reader \& Manipulator provides various options for protein manipulation, such as mutations, different protonation states of titratable residues, disulfide bonds, post-translational modifications (i.e., phosphorylation, glycosylation, and lipid-tail linkers), nucleic acid modifications (e.g., more than 100 naturally occurring modified ribonucleotides), and/or other experimental modifications (e.g., adding spin labels, fluorophores, and unnatural amino acids). We added a "GPI anchor" option, so that users can easily link the GPI moiety to their protein of interest (Figure S4 A). Upon user's activation on "GPI anchor" checkbox, as a default, the GPI core sequence (Man( $(\alpha 1-2) \operatorname{Man}(\alpha 1-6) \operatorname{Man}(\alpha 1-4) \mathrm{GICN}(\alpha 1-6) \mathrm{PI}-\mathrm{DAG}$; Figure S5) is attached to the $\mathrm{C}$-terminus of a protein via ethanolamine phosphate. The user can change the protein chain to be GPI-anchored and also modify the GPI sequence by clicking the "edit" button, which is linked to the GPI-specialized version of Glycolipid Modeler (Figure S4 B), so that glycans and/or chemical modifications to the GPI core sequence can be easily added.

The user-specified GPI sequence is transferred to the server in GRS format and used to generate the GPI structure linked to the protein. PDB Reader \& Manipulator first generates the connectivity information of GPI, and the coordinates of GPI are imported using the GPI core model structure from our previous study ${ }^{65}$, which is pre-optimized and pre-oriented with respect 
to the membrane normal (the $z$ axis) and center $(z=0)$. Some coordinates may not be built during this process due to the absence of the coordinate information in the model structure, mostly when a user modifies the GPI sequence or adds an extra chemical modification(s) in the sequence. In such a case, the missing coordinates are generated from the IC information in the CHARMM36 FF. To avoid the penetration of the non-GPI part of the GPI-anchored protein into the membrane, PDB Reader \& Manipulator searches an appropriate orientation of the protein by changing two dihedral angles in the ethanolamine group ( $\theta$ and $\omega$ in Figure S5). While PDB Reader \& Manipulator exists as an independent module, it is the first step in other CHARMMGUI modules, so that a user can easily build various membrane systems with a GPI-anchored protein.

LPS Modeler. A LPS molecule is specific to the Gram-negative bacteria and consists of three regions: lipid $A$, core oligosaccharide, and O-antigen polysaccharide (Figure 2 A). In most cases, the lipid A contains a $\beta$ - $(1 \rightarrow 6)$-linked glucosamine disaccharide that carries 4 to 7 amideand ester-linked acyl chains anchoring the LPS into the $\mathrm{OM}^{66}$. A core oligosaccharide linked to lipid $A$ is made up of up to 15 monosaccharides that always include the negatively charged eight-carbon sugar 3-deoxy-D-manno-oct-2-ulosonic acid (Kdo), and in many cases, the sevencarbon sugar heptose and common hexose residues (Figure S6 A). Additional decorative groups can be present in the form of amine, phosphate, or ethanolamine diphosphate groups (Figure S6 B-C), which provide charge variability to bacterial membranes. This region varies structurally (e.g., there are five core types, R1-R4 and $\mathrm{K}-12$, in E. coli). The O-antigen polysaccharide is typically attached to the outer part of the core region. Most of these polysaccharides are made of 2 to 8 sugar residues in a repetitive manner, referred to as a repeating unit (RU), which can be polymerized 10 to 100 times. An O-antigen RU can be neutral or with monovalent anionic charges (Figure S6 C). The diversity in sugar and substituent types in the bacterial glycome, the linkage positions, and the extents of polymerization make a LPS structure highly variable and complex. Bacterial growth conditions can influence the expression of surface determinants resulting in the loss or reduction of O-antigen polysaccharides ${ }^{67-71}$. Some bacteria naturally form LPS lacking the O-antigen, while others form a lipid A-core with further sugar modifications (also called LOS). Unlike other typical LPS cores, the LOS core is less negatively charged (Figure $\mathbf{S 6} \mathbf{C}$ ) because the ethanolamine phosphate zwitterion is the most dominant chemical modification in LOS (Figure S6 B).

Similar to Glycolipid Modeler, LPS Modeler has been developed to facilitate the structure generation of a single LPS or LOS molecule. LPS Modeler takes an LPS/LOS sequence from the predefined LPS/LOS sequences as input data, i.e., the user can select the bacterial species, lipid A / core / O-antigen types, and the number of O-antigen RUs (Figure 2 B). Further, available $\mathrm{O}$-antigens for a specific bacterium are provided as a table, so that they can be easily changed by clicking an O-antigen name (Figure 2 B). LPS Modeler currently supports experimentally identified LPS sequences ${ }^{72-76}$ of 15 bacterial species with 37 lipid A, 52 core, and 304 O-antigen types (Table 2), including all available E. coli O-antigen sequences in $\mathrm{ECODAB}^{77,78}$. The predefined LPS/LOS sequences are stored in GRS format on the server, which can be transferred to the client side upon request by the user.

The selected LPS/LOS sequence is automatically displayed in the CASPER format ${ }^{22,79}$ next to the core selection button or O-antigen text input box (Figure 2 B), as well as on the 


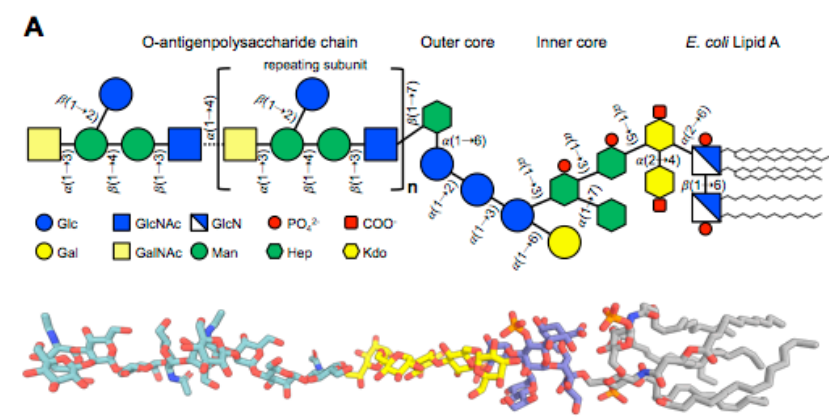

C Core Sequence:

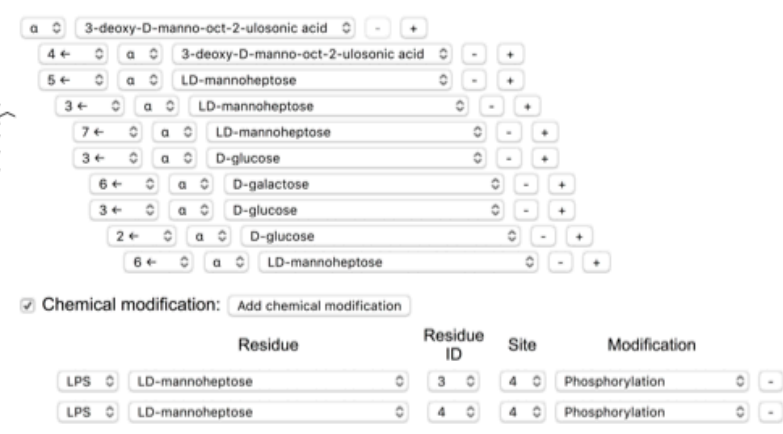

B LPS Sequence:

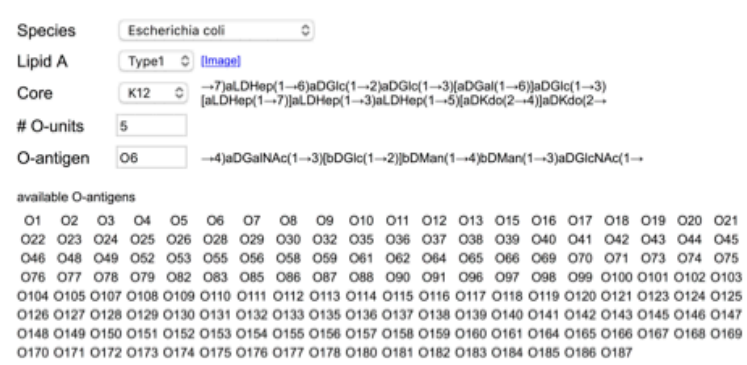

D Core - O-antigen Linkage:

core resid

$1007+0 \beta 3$

\section{E O-antigen Sequence:}

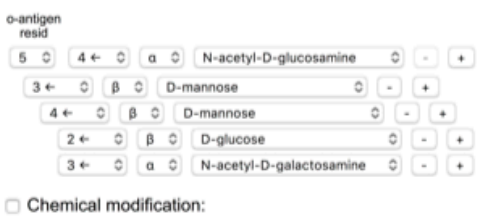

Figure 2. Structure of (A) LPS and (B-E) illustrative snapshots of LPS Modeler. (A) E. coli K-12 O6 with 2 repeating units. (B) Users can build a LPS sequence using bacterial species, lipid $A$, core, and O-antigen information. The selected LPS sequence is displayed on (C) "Core Sequence" and (E) "O-antigen Sequence" panels, and the linkage information between core and O-antigen is displayed in (D) "Core - O-antigen Linkage" section.

"Core Sequence" (Figure 2 C) and "O-antigen Sequence" (Figure 2 E) panels. Using these glycan sequence panels, the user can easily modify the carbohydrate types, linkage types, and chemical modifications, or can add or remove a carbohydrate or a chemical modification to/from the predefined sequence. In addition, with the presence of O-antigen RUs, the information for the core-O-antigen linkage is displayed (Figure 2 D), and the user can readily modify the linkage between core and O-antigen using the selection buttons. If LOS is selected or a user puts zero in the "\# O-units" text box to build rough LPS structure, the "Core-O-antigen Linkage" and "O-antigen Sequence" panels will be hidden to avoid confusion in identifying the selected sequence. Finally, based on reported chemical modifications or acylation patterns, there are more than one lipid A types available in a selected bacterial species, which user can check their chemical structures and choose one of available lipid A structures.

Upon clicking on the next button, LPS Modeler generates a CHARMM input script using user-selected sequence information to build an all-atom LPS/LOS structure. The structure generation procedure in LPS Modeler is rather complicated compared to that in Glycolipid Modeler, because LPS/LOS contains multiple components. In addition, to use the generated LPS/LOS structure for the OM system building, the structure needs to be extended along the membrane normal to avoid any steric clashes with the neighboring LPS/LOS molecules. Therefore, additional procedures are required to make an extended structure by applying planar and cylindrical restraints. The detailed structure generation procedure is as follows: (i) generate connectivity information and coordinates of lipid A using the IC information in the CHARMM36 FF; (ii) reorient the generated lipid $A$ structure to be aligned along the z-axis; (iii) generate connectivity information of core oligosaccharides, make a link to lipid $A$, and build coordinates using the IC information; (iv) generate connectivity information of O-antigen polysaccharides, 
Table 2. Supported bacterial species with the number of lipid A, core, and O-antigen types in LPS Modeler.

\begin{tabular}{lccc}
\hline Bacterial species & \# of Lipid A types & \# of Core types & \# of O-antigen types \\
\hline Acinetobacter baumannii & 2 & 3 & - \\
Burkholderia cepacia & 3 & 1 & - \\
Campylobacter jejuni & 1 & 9 & - \\
Chlamydia trachomatis & 1 & 3 & 157 \\
Escherichia coli & 2 & 5 & 5 \\
Helicobacter pylori & 2 & 1 & 16 \\
Klebsiella pneumonia & 3 & 1 & - \\
Moraxella catarrhalis & 1 & 3 & - \\
Neisseria gonorrhoeae & 3 & 3 & - \\
Neisseria meningitidis & 3 & 8 & 32 \\
Pseudomonas aeruginosa & 5 & 3 & 60 \\
Salmonella enterica & 3 & 2 & 19 \\
Shigella flexneri & 1 & 1 & 15 \\
Vibrio cholerae & 5 & 3 & - \\
Yersinia pestis & 2 & 6 & 304 \\
\hline Total & 37 & 52 & \\
\hline
\end{tabular}

connect the O-antigen to the core, and build coordinates using the IC information; (v) make LPS structure extended by performing a short MD simulation with planar (in z position) and cylindrical restraints (see Figure 2 A for E. coli LPS structure with the K-12 core and the O6 Oantigen generated through this procedure). All the input (CHARMM script) and output (LPS structure, connectivity information, and parameter) files can be downloaded by clicking the "download.tgz" button.

Glycolipid-/LPS-containing Membrane Builder. Although a single glycolipid/LPS/LOS structure can be generated by Glycolipid Modeler and LPS Modeler, building a membrane system containing them is not straightforward. In addition to knowledge of structural models of individual proteins and lipids, handling of all relevant information to correctly prepare a complex membrane system requires considerable time and experience. Membrane Builder successfully encapsulates such a sophisticated process into a well-established five-step workflow, and it can reproducibly generate a realistic protein-membrane or membrane-only system. Therefore, glycolipid-/LPS-containing Membrane Builder is implemented by incorporating Glycolipid Modeler and LPS Modeler into the lipid selection in Membrane Builder. The first step of Membrane Builder is protein structure reading and manipulation, which are managed by $P D B$ Reader \& Manipulator. Introduction of a GPI anchor into the protein can be done with the "GPI anchor" option during this step (Figure S4). In STEP 2, Membrane Builder provides options to change the orientation and position of the protein along the membrane normal. In STEP 3, which is the first step for the membrane-only system, users can select the lipid components, and the incorporation of Glycolipid Modeler and LPS Modeler is implemented in this step. As glycolipid-/LPS-containing Membrane Builder should allow multiple glycolipid/LPS selections, we introduced "Glycolipids" and "LPS (lipopolysaccharides)" subgroups in the lipid selection table of Membrane Builder (Figure 3 A). When the glycolipid and/or LPS section(s) are activated, "Add glycolipid" and/or "Add LPS" buttons will show up. By clinking these buttons, a new glycolipid (default is GalCer) or LPS (default is E. coli with R1 core and O1 O-antigen) molecule is added to the lipid selection table with a sequential ID (starting from GLPA to GLPZZ for glycolipids and from LPSA to LPSZZ for LPS). The glycolipid or LPS ID is implemented as a clickable button, which is linked to a pop-up window that contains Glycolipid Modeler (Figure 3 
B) or LPS Modeler, so that one can easily add a pre-defined or custom glycolipid/LPS/LOS molecule in the selection table. The lipid selection information is used to place pseudo spheres for the lipid head group position, and the pseudo spheres are replaced with the all-atom lipid structures in STEP 4. Other components, such as water and ions, are also generated in STEP 4, and all the components are assembled in STEP 5. The simulation system, topology and parameter files, ready-to-use MD inputs (based upon user's selection), as well as the CHARMM input files used to generate the simulation system are provided as a single tar file ("download.tgz").

Unlike typical phospholipids, glycolipid and LPS molecules require specific considerations for the lipid component generation. (i) While Membrane Builder uses a predetermined set of 2,000 conformations for each phospholipid during the replacement of pseudo spheres, it is impossible to prepare such a conformation library for each glycolipid or LPS/LOS molecule because their carbohydrate sequence and lipid type can be varied depending on the user selection. Therefore, we introduced an additional step to generate a structure for each user-specified glycolipid or LPS/LOS sequence (Figure S7; highlighted in green). The generated glycolipid or LPS/LOS structure is then used to replace the pseudo spheres in STEP 4. (ii) While phospholipids are designed to be a single residue in the CHARMM FF, glycolipid and LPS/LOS molecules are composed of lipid and carbohydrate residues (socalled segment). Therefore, the CHARMM input script to generate a membrane component in STEP 4 was modified to handle both phospholipid residues and glycolipid or LPS/LOS segments (Figure S7; highlighted in orange). (iii) Because some glycolipids, especially LPS, have a very long glycan chain, it can cause severe steric crashes with the neighboring proteins or LPS during the system generation. To avoid this problem, Membrane Builder projects some protein atoms onto the $X Y$ plane at $z= \pm 12 \AA$ to consider the protein shape above and below the membrane. In addition, each glycolipid or LPS/LOS structure is made to be extended along the membrane normal. (iv) The divalent cation provides stability to the bacterial OM by forming

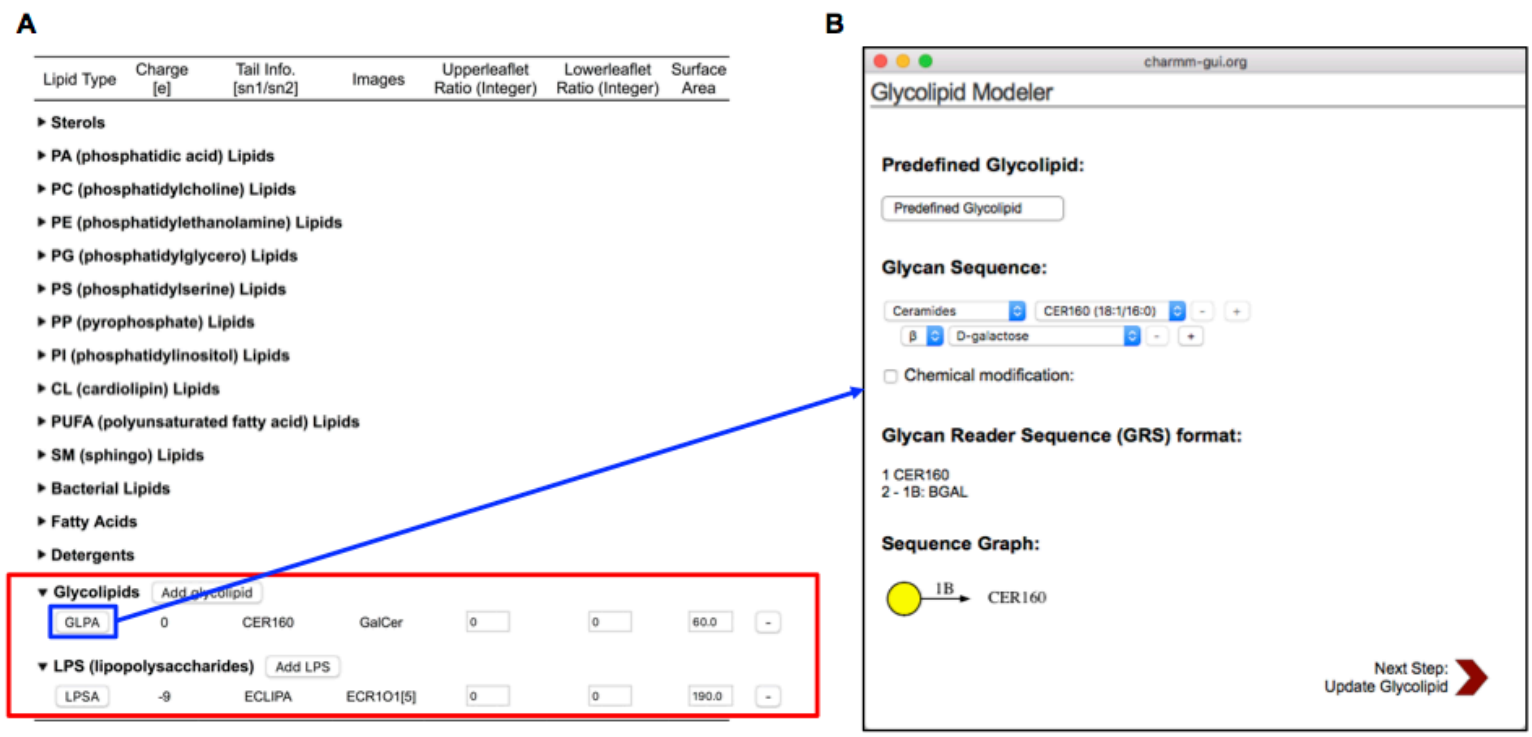

Figure 3. (A) Implementation of "Glycolipids" and "LPS (lipopolysaccharides)" subgroups in the lipid selection table in Membrane Builder (highlighted in red box). The glycolipid ID (highlighted in blue box) is linked to (B) Glycolipid Modeler in a new pop-up window. In the same way, the LPS ID is linked to LPS Modeler, but it is not shown in this figure. 
salt bridges with negatively charged lipid $A$ and core. Therefore, the ion placement method in STEP 4 was modified to place the $\mathrm{Ca}^{2+}$ divalent cations in the lipid $\mathrm{A}$ and core regions as the counter ions. Membrane Builder also provides options to change the type of counter ions for lipid $\mathrm{A}$ and core, so a user can select the ion types among $\mathrm{K}^{+}, \mathrm{Na}^{+}, \mathrm{Mg}^{2+}$, and $\mathrm{Ca}^{2+}$.

\section{RESULTS AND DISCUSSION}

Several membrane-protein complex and membrane-only systems were generated and simulated to illustrate glycolipid-/LPS-containing Membrane Builder in a combination with PDB Reader \& Manipulator. Note that these illustration systems are chosen to show the new capability of Membrane Builder but not for the FF validation or scientific researches. The issue in FF validation is discussed in the LIMITATIONS section below. The video demos of their system building procedures are available on http://www.charmmgui.org/demo/membrane_builder/2. All the prepared simulation systems were triplicated to increase sampling and to check the convergence. All simulations were performed using OpenMM 7.1.1 $1^{39}$ with the OpenMM Python scripts provided by CHARMM-GUI ${ }^{31}$. The simulation systems were minimized and equilibrated following the CHARMM-GUI Membrane Builder standard protocols ${ }^{31}$, and 500 -ns production runs for plasma membrane systems and 1- $\mu$ s production runs for OM systems were carried out in NPT (constant number of atoms and

A

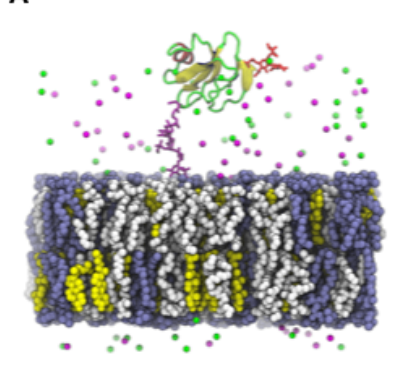

E

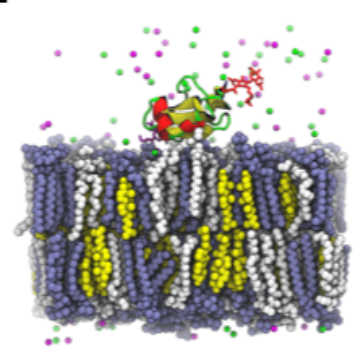

B

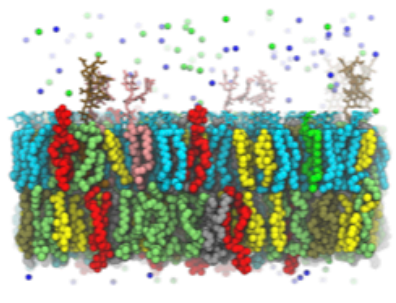

$\mathbf{F}$

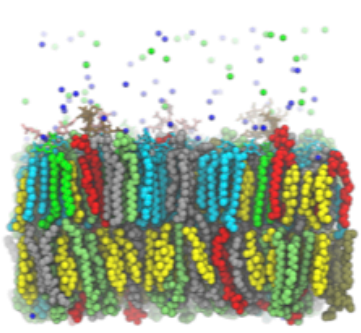

C

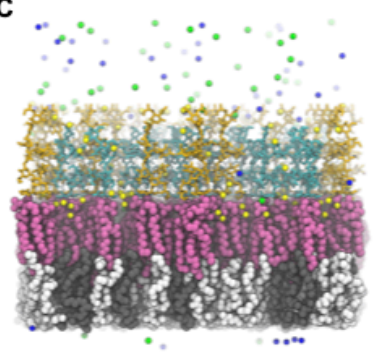

G

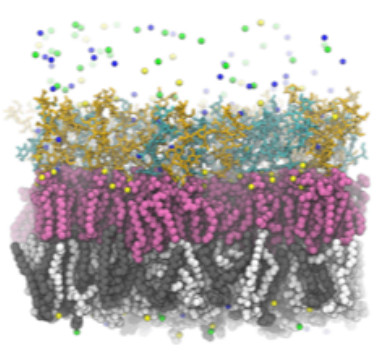

D

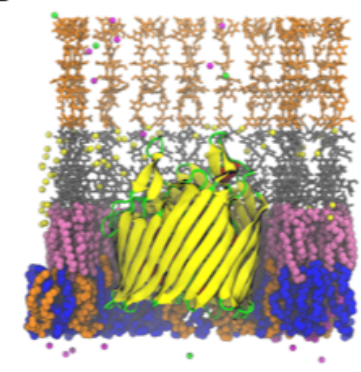

H

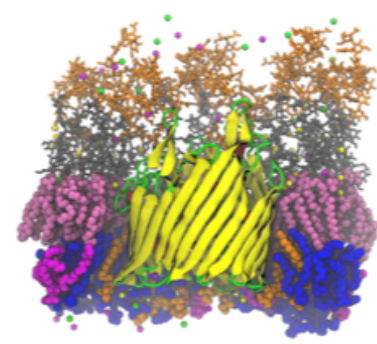

Figure 4. Snapshots of $(A-D)$ initial and $(E-H)$ final simulation systems for $(A, E)$ GPI-CD59, $(B, F)$ axolemma, $(C, G) C$. jejuni $\mathrm{OM}$, and $(\mathrm{D}, \mathrm{H}) \mathrm{BtuB}$ in $E$. coli OM. Proteins are represented in cartoon with different colors based on the secondary structures (red for $\alpha$-helix, yellow for $\beta$-sheet, and green for coil and turn). Carbohydrates are represented in red (N-glycan), sky-blue (GalCer), green (sulfatides), light-pink (GM1), brown (GD1a), cyan (C. jejuni GM1-mimicry LOS), light-orange (C. jejuni GD1a-mimicry LOS), gray (E. coli core), and orange (E. coli O-antigen) sticks. Lipid moieties are showed in yellow (Chol), white (POPE), iceblue (PSM), sky-blue (GalCer), green (sulfatides), coral (GM1), brown (GD1a), lime (POPC), light-gray (SOPE), cyan (SSM), tan (POPS), red (POPI), gray (POPG), blue (PPPE), orange (PVPG), magenta (PVCL2), and pink (E. coli/C. jejuni lipid A) spheres. $\mathrm{Ca}^{2+}, \mathrm{Na}^{+}, \mathrm{K}^{+}$, and $\mathrm{Cl}^{-}$ions are represented in yellow, blue, magenta, and green small spheres, respectively. The water molecules are omitted for clarity. 
isothermal-isobaric) ensemble. The temperature was maintained at $300.0 \mathrm{~K}$ for the GPI-CD59 system and $310.15 \mathrm{~K}$ for other systems, and the pressure was set to $1.0 \mathrm{bar}$.

GPI-anchored CD59 in human plasma membranes. As an example of building a membrane system containing GPI-anchored protein, we built a system of GPI-anchored CD59 in human plasma membranes. The CD59 is a surface-bound complement regulatory protein that protects the cell from reactive lysis by inhibiting the formation of membrane attack complex ${ }^{80}$. The structure of CD59 was obtained from the PDB (ID: 1CDR) ${ }^{43}$, and the GPI structure (Man $(\alpha 1$ 2)Man( $\alpha 1-6)$ Man2EtNP $(\alpha 1-4) \mathrm{GlcN}(\alpha 1-6)$ PI-DAG; EtNP is ethanolamine phosphate $)^{81}$ was linked to the C-terminus of the protein. Note that we only use the well-identified glycans and chemical modifications for the GPI in this study. In addition, an N-glycan in the crystal structure was included. The GPI-anchored CD59 was inserted into a lipid raft model containing 145 cholesterols (Chol), 149 1-palmitoyl-2-oleoyl-sn-phosphatidylethanolamines (POPE), and 135 palmitoyl-sphingomyelins $(\mathrm{PSM})^{65}$. The TIP3P water box was added to the system with $\mathrm{K}^{+}$ counterions and $0.15 \mathrm{M}$ of $\mathrm{K}^{+}$and $\mathrm{Cl}^{-}$ions. Figure $4 \mathrm{~A}$ shows the initial structure of the generated system. After $500 \mathrm{~ns}$, membrane shrank in xy (membrane plane) but extended in $\mathrm{z}$ direction, showing the lipid ordered phase (Figure 4 B). All three replicas were in an equilibrium state within 100 ns of simulation (Figure S8 A).

Axonal membranes in human neuron cells with various gangliosides. The axolemma is the neuron cell membrane surrounding an axon, maintaining the concentration gradient of ions inside and outside of the axon, which is essential for the signal transmission. Although the lipid composition of axolemma was identified ${ }^{82}$, the lipid composition in each leaflet is still elusive because a certain lipid type only exists in the outer leaflet. For example, the axonal membranes contain several gangliosides, such as GM1, GD1a, and GQ1b, as well as GalCer and sulfatides in their outer leaflet. To get the accurate lipid composition in each leaflet, we have performed testing simulations of symmetric bilayers: one with the estimated number of inner leaflet components (99 Chol, 39 POPC, 38 SOPE, 6 SSM, 6 POPS, and 8 POPI), and the other with the estimated number of outer leaflet components (50 Chol, 20 POPC, 19 SOPE, 3 SSM, 3 POPS, 4 POPI, 69 GalCer, 14 sulfatides, and 6 gangliosides), where the abbreviations are POPC for 1-palmitoyl-2-oleoyl-sn-phosphatidylcholines, SOPE for 1-stearoyl-2-oleoyl-snphosphatidylethanolamines, SSM for stearoyl-sphingomyelins, POPS for 1-palmitoyl-2-oleoylsn-phosphatidylserines, and POPI for 1-palmitoyl-2-oleoyl-sn-phosphatidylinositols. Using the area-per-lipid (APL) data obtained from the testing simulations, the accurate lipid composition was calculated; see Section S1 in the supporting materials for details. Finally, an asymmetric bilayer system was built with 119 Chol, 47 POPC, 46 SOPE, 7 SSM, 7 POPS, and 10 POPI in the inner leaflet, and with 66 Chol, 26 POPC, 25 SOPE, 4 SSM, 4 POPS, 5 POPI, 86 GalCer, 17 sulfatides, $4 \mathrm{GM} 1$, and $4 \mathrm{GD} 1 \mathrm{a}$ in the outer leaflet. The TIP3P water box, $\mathrm{Na}^{+}$counterions, and $0.15 \mathrm{M}$ of $\mathrm{Na}^{+}$and $\mathrm{Cl}^{-}$ions were added to the system. Like GPI-CD59 system, the axolemma system showed the phase transition from the lipid disordered phase (Figure $4 \mathbf{B}$ ) to the lipid ordered phase after 500-ns simulation (Figure 4 F). This is consistent with the previous researches $^{83,84}$ showing the high concentration of Chol and SM results in lipid ordered phase. The system size in $X / Y$ and $Z$ was converged within 100 ns (Figure S8 B), implying the fast mobility of phospholipids. 
C. jejuni OM. C. jejuni has ganglioside-mimicry LOS on its OM. Building C. jejuni OM models was more straightforward than building axolemma models because the outer leaflet of $C$. jejuni OM only contains ganglioside-mimicry LOS, and the inner leaflet consists of 8:2 mixture of POPE and 1-palmitoyl-2-oleoyl-sn-phosphatidylglycerol (POPG $)^{85}$. Although the lipid composition of each leaflet is clearly identified, the ratio between the LOS in the outer leaflet and the phospholipids in the inner leaflet is unknown. Therefore, we tested symmetric bilayer simulations of the outer leaflet component systems (GM1-mimicry LOS and GD1a-mimicry LOS) and the inner leaflet component system (POPE:POPG $=8: 2$ ), and calculated the number of lipids in each leaflet by measuring the APL of each system (Table S2). Finally, an asymmetric bilayer system was built with 138 POPE and 34 POPG in the inner leaflet, and 30 GM1-mimicry LOS and 30 GD1a-mimicry LOS in the outer leaflet. The lipid $A$ and core were neutralized with $\mathrm{Ca}^{2+}$ ions, and $\mathrm{Na}^{+}$ions were added to neutralize phospholipids in the inner leaflet. In addition,

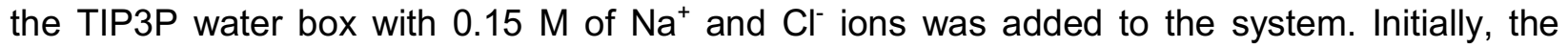
oligosaccharides of LOS were extended along the membrane normal (Figure $4 \mathrm{C}$ ), and they were relaxed after 500 -ns simulation (Figure $\mathbf{4} \mathbf{G}$ ). The system size was converged about after 700-ns simulation (Figure S8 C).

BtuB in E. coli OM. BtuB is a TonB-dependent E. coli transporter for cobalamin (Vitamin $\mathrm{B}_{12}$ ). A BtuB-containing $E$. coli OM system was generated to illustrate the generation of OM system containing OM protein. The pre-oriented BtuB structure (PDB ID: $1 \mathrm{NQE})^{48}$ was obtained from OPM database ${ }^{86} .75$ PPPE, 20 PVPG, and 5 PVCL2 were added to the inner leaflet, and $38 \mathrm{E}$. coli LPS structures with the K-12 core and the O6 O-antigen with 2 RUs were added to the outer leaflet following the procedure described in the references ${ }^{87,88}$; abbreviations are PPPE for 1palmitoyl-2-palmitoleoyl-sn-phosphatidylethanolamine, PVPG for 1-palmitoyl-2-vacenoyl-snphosphatidylglycerol, and PVCL2 for 1,1'-palmitoyl-2,2'-vacenoyl cardiolipin with a net charge of $-2 \mathrm{e}$. The TIP3P water, $\mathrm{K}^{+}$counterions, and $0.15 \mathrm{M} \mathrm{of} \mathrm{K}^{+}$and $\mathrm{Cl}^{-}$ions were added to the system. Like C. jejuni OM system, E. coli core and O-antigen were initially extended along the membrane normal (Figure $4 \mathrm{D}$ ), and they were relaxed after 500-ns simulation (Figure $4 \mathrm{H}$ ). Although 2 O-antigen RUs were used in this study, it was sufficient to hide the vestibule of BtuB (Figure S9). While the plasma membrane systems (GPI-CD59 and axolemma) were stabilized within 100-ns simulation (Figure S8 A-B), the bacterial OM systems (C. jejuni OM and BtuB in E. coli OM) required longer simulation time (> $700 \mathrm{~ns}$ ) to be stabilized (Figure S8 C-D). This is consistent with previous computational OM studies ${ }^{87-89}$, and it is believed that strong interaction between LPS and divalent cation causes this phenomenon.

Limitations. Although CHARMM-GUI Membrane Builder is robust, for its successful practical use, there are some limitations that users need to be aware of. As we described in BtuB in $E$. coli OM subsection in the RESULTS AND DISCUSSION section, the membrane dynamics is very slow especially for Gram-negative bacterial OM systems due to the slow diffusion of the LPS molecule. Since CHARMM-GUI Membrane Builder provides only initial simulation systems, a user needs to perform a long simulation ( $>400 \mathrm{~ns}$ ) to equilibrate the system if it contains LPS molecules. 
In most cases, experiments provide the overall lipid composition, but one has to determine how many lipids should be in each leaflet for their simulation. This issue is relatively simpler for a symmetric bilayer system (i.e., the same lipid compositions for both the inner and outer leaflets). However, it becomes complicated to estimate the number of lipids in each leaflet for an asymmetric system like an OM. To avoid an asymmetric lateral pressure of a bilayer membrane, a current best solution, also used in Membrane Builder, is to determine and distribute necessary number of each lipid type in the inner and outer leaflets to have the same membrane surface area in each leaflet. One can simply use the area-per-lipid estimated from homogeneous lipid bilayer systems. Alternatively, one can build and simulate two symmetrical bilayer systems: one with the inner leaflet composition and the other with the outer leaflet composition. By doing this, the user can get accurate area-per-lipid data from these test simulations, and use them in Membrane Builder to get more accurate number of each lipid type in the system (see Section $\mathbf{S 1}$ in the supporting materials and video demos on http://www.charmm-gui.org/demo/membrane_builder/3).

CHARMM-GUI Membrane Builder uses the latest version of the CHARMM C36 additive $\mathrm{FF}^{61-63}$. Similar to other FFs, the CHARMM FF is designed to allow a "building-block" approach to easily create FFs for molecules analogous to the ones already in the FF. Many topologies and parameters of lipids and carbohydrates in the latest version of the CHARMM C36 FF were generated using this "building-block" approach, and the generated FFs were further validated for several years by comparing the simulations with the available experimental data ${ }^{65,66,87,88,90-99}$.

\section{CONCLUSIONS}

The new features we introduced in CHARMM-GUI (Glycolipid Modeler, LPS Modeler, and glycolipid-/LPS-containing Membrane Builder) make it possible to generate a realistic biomembrane simulation system containing glycolipids and/or LPS/LOS. Glycolipid Modeler and LPS Modeler can not only provide the glycolipid/LPS/LOS structures, but also be utilized as a database. In other words, they provide an easy way to navigate glycolipid structures in a certain glycolipid class, comparing the glycolipid structure of interest with other glycolipids in the same category, or comparing certain LPS/LOS structure or sequence with those of other bacterial strains or species. Using four biological membrane systems, we have also illustrated the generated systems through glycolipid-/LPS-containing Membrane Builder are reliable. These tools will help carrying out innovative and novel glycolipid/LPS/LOS modeling and simulation research to acquire insight into structures, dynamics, and underlying mechanisms of complex glycolipid-/LPS-/LOS-containing systems.

\section{ACKNOWLEDGEMENTS}

This work was supported in part by grants from NSF MCB-1727508, DBI-1707207, DBI1660380, IIA-1664696, NIH U54 GM087519 and R01 GM103695, XSEDE MCB070009 (WI), NSF MCB-1149187 (JBK), and the Swedish Research Council no. 2013-4859 and 2017-03703 (GW). 


\section{SUPPORTING INFORMATION}

Supplementary Methods S1. Detailed methods for axonal membrane system preparation. Table S1. Available lipid and carbohydrate types and chemical modifications in Glycolipid Modeler. Table S2. Calculated lipid composition for $C$. jejuni OM systems. Table S3. Calculating the number of lipids in each leaflet for axolemma system. Table S4. Final lipid composition for axolemma system. Figure $\mathrm{S} 1$. Schematic representations of major core glycan sequences of glycosphingolipids (GSLs). Figure S2. Schematic representations of the glycan motifs for ABO, $P$, and Lewis blood group systems. Figure S3. A part of the CHARMM input script generated for GM1. Figure S4. Implementation of "GPI anchor" in CHARMM-GUI PDB Reader \& Manipulator. Figure S5. GPI core structure. Figure S6. Statistics of LPS sequences from the LPS Modeler predefined sequence library. Figure S7. The workflow of glycolipid-/LPS-containing Membrane Builder. Figure S8. Time series of the system size for each illustrative system. Figure S9. Top view of initial and final BtuB in an E. coli OM. This material is available free of charge via the Internet at http://pubs.acs.org.

\section{NOTES}

The authors declare no competing financial interest. 


\section{REFERENCES}

(1) Sezgin, E.; Levental, I.; Mayor, S.; Eggeling, C. The Mystery of Membrane Organization: Composition, Regulation and Roles of Lipid Rafts. Nat. Rev. Mol. Cell Biol. 2017, 18 (6), 361-374.

(2) Okuda, S.; Sherman, D. J.; Silhavy, T. J.; Ruiz, N.; Kahne, D. Lipopolysaccharide Transport and Assembly at the Outer Membrane: The PEZ Model. Nat. Rev. Microbiol. 2016, 14 (6), 337-345.

(3) Ariga, T.; Yu, R. K. The Role of Globo-Series Glycolipids in Neuronal Cell Differentiation-A Review. Neurochem. Res. 1998, 23 (3), 291-303.

(4) Furukawa, K.; Okuda, T.; Furukawa, K. Roles of Glycolipids in the Development and Maintenance of Nervous Tissues. Methods Enzymol. 2006, 417, 37-52.

(5) Malhotra, R. Membrane Glycolipids: Functional Heterogeneity: A Review. Biochem. Anal. Biochem. 2012, 1 (2), 1000108.

(6) Yamakawa, T.; Nagai, Y. Glycolipids at the Cell Surface and Their Biological Functions. Trends Biochem. Sci. 1978, 3 (2), 128-131.

(7) Holst, O.; Moran, A. P.; Brennan, P. J. Overview of the Glycosylated Components of the Bacterial Cell Envelope. In Microbial Glycobiology; Elsevier, 2010; pp 1-13.

(8) Chester, M. A. Nomenclature of Glycolipids (IUPAC Recommendations 1997). Pure Appl. Chem. 1997, 69 (12), 2475-2487.

(9) Kuwabara, S.; Yuki, N. Axonal Guillain-Barré Syndrome: Concepts and Controversies. Lancet Neurol. 2013, 12 (12), 1180-1188.

(10) Kusunoki, S.; Kaida, K. Antibodies against Ganglioside Complexes in Guillain-Barré Syndrome and Related Disorders. J. Neurochem. 2011, 116 (5), 828-832.

(11) Mondal, S.; Mukhopadhyay, C. Molecular Level Investigation of Organization in Ternary Lipid Bilayer: A Computational Approach. Langmuir 2008, 24 (18), 10298-10305.

(20) Woods, R.; Xue, X.; Davis, R.; Kim, H.; Li, Q.; Nagarajan, M. (Akila); Wang, Y. GLYCAM Web http://glycam.org.

(21) Engelsen, S. B.; Hansen, P. I.; Perez, S. POLYS 2.0: An Open Source Software Package 
for Building Three-Dimensional Structures of Polysaccharides. Biopolymers 2014, 101 (7), 733-743.

(22) Kuttel, M. M.; Stahle, J.; Widmalm, G. CarbBuilder: Software for Building Molecular Models of Complex Oligo- and Polysaccharide Structures. J. Comput. Chem. 2016, 37 (22), 2098-2105.

(23) Danne, R.; Poojari, C.; Martinez-Seara, H.; Rissanen, S.; Lolicato, F.; Róg, T.; Vattulainen, I. DoGlycans -Tools for Preparing Carbohydrate Structures for Atomistic Simulations of Glycoproteins, Glycolipids, and Carbohydrate Polymers for GROMACS. J. Chem. Inf. Model. 2017, 57 (10), 2401-2406.

(24) Martínez, L.; Andrade, R.; Birgin, E. G.; Martínez, J. M. PACKMOL: A Package for Building Initial Configurations for Molecular Dynamics Simulations. J. Comput. Chem. 2009, 30 (13), 2157-2164.

(25) Sommer, B.; Dingersen, T.; Gamroth, C.; Schneider, S. E.; Rubert, S.; Krüger, J.; Dietz, K.-J. CELLmicrocosmos 2.2 MembraneEditor: A Modular Interactive Shape-Based Software Approach To Solve Heterogeneous Membrane Packing Problems. J. Chem. Inf. Model. 2011, 51, 1165-1182.

(26) Wassenaar, T. A.; Ingolfsson, H. I.; Bockmann, R. A.; Tieleman, D. P.; Marrink, S. J. Computational Lipidomics with Insane: A Versatile Tool for Generating Custom Membranes for Molecular Simulations. J. Chem. Theory Comput. 2015, 11 (5), 2144 2155.

(27) Jo, S.; Kim, T.; Iyer, V. G.; Im, W. CHARMM-GUI: A Web-Based Graphical User Interface for CHARMM. J. Comput. Chem. 2008, 29 (11), 1859-1865.

(28) Jo, S.; Kim, T.; Im, W. Automated Builder and Database of Protein/Membrane Complexes for Molecular Dynamics Simulations. PLoS One 2007, 2 (9), e880.

(29) Jo, S.; Lim, J. B.; Klauda, J. B.; Im, W. CHARMM-GUI Membrane Builder for Mixed Bilayers and Its Application to Yeast Membranes. Biophys. J. 2009, 97 (1), 50-58.

(30) Wu, E. L.; Cheng, X.; Jo, S.; Rui, H.; Song, K. C.; Davila-Contreras, E. M.; Qi, Y.; Lee, J.; Monje-Galvan, V.; Venable, R. M.; et al. CHARMM-GUI Membrane Builder toward Realistic Biological Membrane Simulations. J. Comput. Chem. 2014, 35 (27), 1997-2004. Lee, J.; Cheng, X.; Swails, J. M.; Yeom, M. S.; Eastman, P. K.; Lemkul, J. A.; Wei, S.; Buckner, J.; Jeong, J. C.; Qi, Y.; et al. CHARMM-GUI Input Generator for NAMD, GROMACS, AMBER, OpenMM, and CHARMM/OpenMM Simulations Using the CHARMM36 Additive Force Field. J. Chem. Theory Comput. 2016, 12 (1), 405-413. Brooks, B. R.; Brooks 3rd, C. L.; MacKerell Jr., A. D.; Nilsson, L.; Petrella, R. J.; Roux, B.; Won, Y.; Archontis, G.; Bartels, C.; Boresch, S.; et al. CHARMM: The Biomolecular Simulation Program. J. Comput. Chem. 2009, 30 (10), 1545-1614.

Phillips, J. C.; Braun, R.; Wang, W.; Gumbart, J.; Tajkhorshid, E.; Villa, E.; Chipot, C.; Skeel, R. D.; Kale, L.; Schulten, K. Scalable Molecular Dynamics with NAMD. J. Comput. Chem. 2005, 26 (16), 1781-1802.

Abraham, M. J.; Murtola, T.; Schulz, R.; Páll, S.; Smith, J. C.; Hess, B.; Lindahl, E. GROMACS: High Performance Molecular Simulations through Multi-Level Parallelism from Laptops to Supercomputers. SoftwareX 2015, 1-2, 19-25.

Case, D. A.; Cheatham 3rd, T. E.; Darden, T.; Gohlke, H.; Luo, R.; Merz Jr., K. M.; Onufriev, A.; Simmerling, C.; Wang, B.; Woods, R. J. The Amber Biomolecular Simulation Programs. J. Comput. Chem. 2005, 26 (16), 1668-1688.

Jung, J.; Mori, T.; Kobayashi, C.; Matsunaga, Y.; Yoda, T.; Feig, M.; Sugita, Y. GENESIS: A Hybrid-Parallel and Multi-Scale Molecular Dynamics Simulator with Enhanced Sampling Algorithms for Biomolecular and Cellular Simulations. WIREs Comput. Mol. Sci. 2015, 5 (4), 310-323.

Plimpton, S. Fast Parallel Algorithms for Short-Range Molecular Dynamics. J. Comput. Phys. 1995, 117 (1), 1-19. 
(38) Bowers, K. J.; Sacerdoti, F. D.; Salmon, J. K.; Shan, Y.; Shaw, D. E.; Chow, E.; Xu, H.; Dror, R. O.; Eastwood, M. P.; Gregersen, B. A.; et al. Scalable Algorithms for Molecular Dynamics Simulations on Commodity Clusters. In The ACM/IEEE Conference on Supercomputing (SC06); Tampa, Florida, 2006.

(39) Eastman, P.; Swails, J.; Chodera, J. D.; McGibbon, R. T.; Zhao, Y.; Beauchamp, K. A.; Wang, L.-P.; Simmonett, A. C.; Harrigan, M. P.; Stern, C. D.; et al. OpenMM 7: Rapid Development of High Performance Algorithms for Molecular Dynamics. PLOS Comput. Biol. 2017, 13 (7), e1005659.

(40) Arthur, E. J.; Brooks 3rd, C. L. Parallelization and Improvements of the Generalized Born Model with a Simple SWitching Function for Modern Graphics Processors. J. Comput. Chem. 2016, 37 (10), 927-939.

(41) Jo, S.; Song, K. C.; Desaire, H.; MacKerell Jr., A. D.; Im, W. Glycan Reader: Automated Sugar Identification and Simulation Preparation for Carbohydrates and Glycoproteins. J. Comput. Chem. 2011, 32 (14), 3135-3141.

(42) Park, S.-J.; Lee, J.; Patel, D. S.; Ma, H.; Lee, H. S.; Jo, S.; Im, W. Glycan Reader Is Improved to Recognize Most Sugar Types and Chemical Modifications in the Protein Data Bank. Bioinformatics 2017, 33 (19), 3051-3057.

(43) Fletcher, C. M.; Harrison, R. A.; Lachmann, P. J.; Neuhaus, D. Structure of a Soluble, Glycosylated Form of the Human Complement Regulatory Protein CD59. Structure 1994, 2 (3), 185-199.

(44) Svennerholm, L.; Boström, K.; Fredman, P.; Jungbjer, B.; Lekman, A.; Månsson, J.-E.; Rynmark, B.-M. Gangliosides and Allied Glycosphingolipids in Human Peripheral Nerve and Spinal Cord. Biochim. Biophys. Acta - Lipids Lipid Metab. 1994, 1214 (2), 115-123. Svennerholm, L.; Boström, K.; Fredman, P.; Jungbjer, B.; Månsson, J.-E.; Rynmark, B.M. Membrane Lipids of Human Peripheral Nerve and Spinal Cord. Biochim. Biophys. Acta - Lipids Lipid Metab. 1992, 1128 (1), 1-7.

(46) MORAN, A. P.; ZAHRINGER, U.; SEYDEL, U.; SCHOLZ, D.; STUTZ, P.; RIETSCHEL, E. T. Structural Analysis of the Lipid A Component of Campylobacter Jejuni CCUG 10936 (Serotype O:2) Lipopolysaccharide. Eur. J. Biochem. 1991, 198 (2), 459-469.

(47) Wilkinson, S. G. Bacterial Lipopolysaccharides-Themes and Variations. Prog. Lipid Res. 1996, 35 (3), 283-343.

(48) Chimento, D. P.; Mohanty, A. K.; Kadner, R. J.; Wiener, M. C. Substrate-Induced Transmembrane Signaling in the Cobalamin Transporter BtuB. Nat. Struct. Mol. Biol. 2003, 10 (5), 394-401.

(49) Cummings, R. D. The Repertoire of Glycan Determinants in the Human Glycome. Mol. Biosyst. 2009, 5 (10), 1087-1104.

(50) Ferguson, M. A. J.; Kinoshita, T.; Hart, G. W. Glycosylphosphatidylinositol Anchors. In Essentials of Glycobiology; Varki, A., Cummings, R. D., Esko, J. D., Freeze, H. H., Stanley, P., Bertozzi, C. R., Hart, G. W., Etzler, M. E., Eds.; Cold Spring Harbor Laboratory Press: Cold Spring Harbor, New York, 2009.

(51) Merrill Jr., A. H.; Park, M.; Koppikar, A.; Lee, R.; Suzuki, A.; Uyesugi, K.; Wang, J.; Wang, M. D. SphinGOMAP http://www.sphingomap.org.

(52) Sonnino, S.; Mauri, L.; Chigorno, V.; Prinetti, A. Gangliosides as Components of Lipid Membrane Domains. Glycobiology 2007, 17 (1), 1R-13R.

(53) Hölzl, G.; Dörmann, P. Structure and Function of Glycoglycerolipids in Plants and Bacteria. Prog. Lipid Res. 2007, 46 (5), 225-243.

(54) Orlean, P.; Menon, A. K. Thematic Review Series: Lipid Posttranslational Modifications. GPI Anchoring of Protein in Yeast and Mammalian Cells, or: How We Learned to Stop Worrying and Love Glycophospholipids. J. Lipid Res. 2007, 48 (5), 993-1011.

(55) Udenfriend, S.; Kodukula, K. How Glycosylphosphatidylinositol-Anchored Membrane Proteins Are Made. Annu. Rev. Biochem. 1995, 64, 563-591. 
(56) McConville, M. J.; Ferguson, M. A. J. The Structure, Biosynthesis and Function of Glycosylated Phosphatidylinositols in the Parasitic Protozoa and Higher Eukaryotes.

Biochem. J. 1993, 294 (2), 305-324.

(57) Storry, J. R.; Castilho, L.; Chen, Q.; Daniels, G.; Denomme, G.; Flegel, W. A.; Gassner, C.; de Haas, M.; Hyland, C.; Keller, M.; et al. International Society of Blood Transfusion Working Party on Red Cell Immunogenetics and Terminology: Report of the Seoul and London Meetings. ISBT Sci. Ser. 2016, 11 (2), 118-122.

(58) Honke, K. Biosynthesis and Biological Function of Sulfoglycolipids. Proc. Japan Acad. Ser. B 2013, 89 (4), 129-138.

(59) Eckhardt, M. The Role and Metabolism of Sulfatide in the Nervous System. Mol. Neurobiol. 2008, 37 (2-3), 93-103.

(60) Varki, A.; Cummings, R. D.; Aebi, M.; Packer, N. H.; Seeberger, P. H.; Esko, J. D.; Stanley, P.; Hart, G.; Darvill, A.; Kinoshita, T.; et al. Symbol Nomenclature for Graphical Representations of Glycans. Glycobiology 2015, 25 (12), 1323-1324.

(61) Klauda, J. B.; Venable, R. M.; Freites, J. A.; O’Connor, J. W.; Tobias, D. J.; MondragonRamirez, C.; Vorobyov, I.; MacKerell Jr., A. D.; Pastor, R. W. Update of the CHARMM All-Atom Additive Force Field for Lipids: Validation on Six Lipid Types. J. Phys. Chem. B 2010, 114 (23), 7830-7843.

(62) Venable, R. M.; Sodt, A. J.; Rogaski, B.; Rui, H.; Hatcher, E.; MacKerell Jr., A. D.; Pastor, R. W.; Klauda, J. B. CHARMM All-Atom Additive Force Field for Sphingomyelin: Elucidation of Hydrogen Bonding and of Positive Curvature. Biophys. J. 2014, 107 (1), 134-145.

(63) Guvench, O.; Greene, S. N.; Kamath, G.; Brady, J. W.; Venable, R. M.; Pastor, R. W.; MacKerell Jr., A. D. Additive Empirical Force Field for Hexopyranose Monosaccharides. J. Comput. Chem. 2008, 29 (15), 2543-2564.

(64) Jo, S.; Cheng, X.; Islam, S. M.; Huang, L.; Rui, H.; Zhu, A.; Lee, H. S.; Qi, Y.; Han, W.; Vanommeslaeghe, K.; et al. CHARMM-GUI PDB Manipulator for Advanced Modeling and Simulations of Proteins Containing Nonstandard Residues. Adv. Protein Chem. Struct. Biol. 2014, 96, 235-265.

(65) Wu, E. L.; Qi, Y.; Park, S.; Mallajosyula, S. S.; MacKerell Jr., A. D.; Klauda, J. B.; Im, W. Insight into Early-Stage Unfolding of GPI-Anchored Human Prion Protein. Biophys. J. 2015, 109 (10), 2090-2100.

(66) Kim, S.; Patel, D. S.; Park, S.; Slusky, J.; Klauda, J. B.; Widmalm, G.; Im, W. Bilayer Properties of Lipid A from Various Gram-Negative Bacteria. Biophys. J. 2016, 111 (8), 1750-1760.

(67) Osawa, K.; Shigemura, K.; Iguchi, A.; Shirai, H.; Imayama, T.; Seto, K.; Raharjo, D.; Fujisawa, M.; Osawa, R.; Shirakawa, T. Modulation of O-antigen Chain Length by the Wzz Gene in Escherichia Coli 0157 Influences Its Sensitivities to Serum Complement. Microbiol. Immunol. 2013, 57 (9), 616-623.

(68) Maldonado, R. F.; Sá-Correia, I.; Valvano, M. A. Lipopolysaccharide Modification in Gram-Negative Bacteria during Chronic Infection. FEMS Microbiol. Rev. 2016, 40 (4), 480-493.

(69) Marvasi, M.; Cox, C. E.; Xu, Y.; Noel, J. T.; Giovannoni, J. J.; Teplitski, M. Differential Regulation of Salmonella Typhimurium Genes Involved in O-Antigen Capsule Production and Their Role in Persistence Within Tomato Fruit. Mol. Plant-Microbe Interact. 2013, 26 (7), 793-800.

(70) Farizano, J. V.; Pescaretti, M. de las M.; López, F. E.; Hsu, F.-F.; Delgado, M. A. The PmrAB System-Inducing Conditions Control Both Lipid A Remodeling and O-Antigen Length Distribution, Influencing the Salmonella Typhimurium-Host Interactions. J. Biol. Chem. 2012, 287 (46), 38778-38789.

(71) Bravo, D.; Silva, C.; Carter, J. A.; Hoare, A.; Alvarez, S. A.; Blondel, C. J.; Zaldivar, M.; 
Valvano, M. A.; Contreras, I. Growth-Phase Regulation of Lipopolysaccharide O-Antigen Chain Length Influences Serum Resistance in Serovars of Salmonella. J. Med. Microbiol. 2008, 57 (8), 938-946.

(72) Knirel, Y. A.; Valvano, M. A. Bacterial Lipopolysaccharides; Knirel, Y. A., Valvano, M. A., Eds.; Springer Vienna: Vienna, 2011.

(73) Raetz, C. R. H.; Whitfield, C. Lipopolysaccharide Endotoxins. Annu. Rev. Biochem. 2002, 71 (1), 635-700.

(74) Liu, B.; Knirel, Y. A.; Feng, L.; Perepelov, A. V.; Senchenkova, S. N.; Reeves, P. R.; Wang, L. Structural Diversity in Salmonella O Antigens and Its Genetic Basis. FEMS Microbiol. Rev. 2014, 38 (1), 56-89.

(75) Kosma, P. Chlamydial Lipopolysaccharide. Biochim. Biophys. Acta - Mol. Basis Dis. 1999, 1455 (2-3), 387-402.

(76) van Vliet, S. J.; Steeghs, L.; Bruijns, S. C. M.; Vaezirad, M. M.; Snijders Blok, C.; Arenas Busto, J. A.; Deken, M.; van Putten, J. P. M.; van Kooyk, Y. Variation of Neisseria Gonorrhoeae Lipooligosaccharide Directs Dendritic Cell-Induced T Helper Responses. PLoS Pathog. 2009, 5 (10), e1000625.

(77) Stenutz, R.; Weintraub, A.; Widmalm, G. The Structures of Escherichia Coli OPolysaccharide Antigens. FEMS Microbiol. Rev. 2006, 30 (3), 382-403.

(78) Rojas-Macias, M. A.; Stahle, J.; Lutteke, T.; Widmalm, G. Development of the ECODAB into a Relational Database for Escherichia Coli O-Antigens and Other Bacterial Polysaccharides. Glycobiology 2015, 25 (3), 341-347.

(79) Lundborg, M.; Widmalm, G. Structural Analysis of Glycans by NMR Chemical Shift Prediction. Anal. Chem. 2011, 83 (5), 1514-1517.

(80) Huang, Y.; Qiao, F.; Abagyan, R.; Hazard, S.; Tomlinson, S. Defining the CD59-C9 Binding Interaction. J. Biol. Chem. 2006, 281 (37), 27398-27404.

(81) Rudd, P. M.; Morgan, B. P.; Wormald, M. R.; Harvey, D. J.; van den Berg, C. W.; Davis, S. J.; Ferguson, M. A. J.; Dwek, R. A. The Glycosylation of the Complement Regulatory Protein, Human Erythrocyte CD59. J. Biol. Chem. 1997, 272 (11), 7229-7244.

(82) DeVries, G. H.; Zetusky, W. J.; Zmachinski, C.; Calabrese, V. P. Lipid Composition of Axolemma-Enriched Fractions from Human Brains. J. Lipid Res. 1981, 22 (2), 208-216. Marsh, D. Liquid-Ordered Phases Induced by Cholesterol: A Compendium of Binary Phase Diagrams. Biochim. Biophys. Acta - Biomembr. 2010, 1798 (3), 688-699. de Almeida, R. F. M.; Fedorov, A.; Prieto, M. Sphingomyelin/Phosphatidylcholine/Cholesterol Phase Diagram: Boundaries and Composition of Lipid Rafts. Biophys. J. 2003, 85 (4), 2406-2416.

(85) Leach, S.; Harvey, P.; Wait, R. Changes with Growth Rate in the Membrane Lipid Composition of and Amino Acid Utilization by Continuous Cultures of Campylobacter Jejuni. J. Appl. Microbiol. 1997, 82 (5), 631-640.

(86) Lomize, M. A.; Pogozheva, I. D.; Joo, H.; Mosberg, H. I.; Lomize, A. L. OPM Database and PPM Web Server: Resources for Positioning of Proteins in Membranes. Nucleic Acids Res. 2012, 40 (D1), D370-D376.

(87) Wu, E. L.; Fleming, P. J.; Yeom, M. S.; Widmalm, G.; Klauda, J. B.; Fleming, K. G.; Im, W. E. Coli Outer Membrane and Interactions with OmpLA. Biophys. J. 2014, 106 (11), 2493-2502.

(88) Patel, D. S.; Re, S.; Wu, E. L.; Qi, Y.; Klebba, P. E.; Widmalm, G.; Yeom, M. S.; Sugita, Y.; Im, W. Dynamics and Interactions of OmpF and LPS: Influence on Pore Accessibility and Ion Permeability. Biophys. J. 2016, 110 (4), 930-938.

(89) Piggot, T. J.; Holdbrook, D. A.; Khalid, S. Electroporation of the E. Coli and S. Aureus Membranes: Molecular Dynamics Simulations of Complex Bacterial Membranes. J. Phys. Chem. B 2011, 115 (45), 13381-13388.

(90) Wu, E. L.; Engstrom, O.; Jo, S.; Stuhlsatz, D.; Yeom, M. S.; Klauda, J. B.; Widmalm, G.; 
Im, W. Molecular Dynamics and NMR Spectroscopy Studies of E. Coli

Lipopolysaccharide Structure and Dynamics. Biophys. J. 2013, 105 (6), 1444-1455.

(91) Jo, S.; Wu, E. L.; Stuhlsatz, D.; Klauda, J. B.; MacKerell, A. D.; Widmalm, G.; Im, W. Lipopolysaccharide Membrane Building and Simulation. In Methods in Molecular Biology: Glycoinformatics; Lütteke, T., Frank, M., Eds.; Humana Press: New York, 2015; pp 391406.

(92) Fleming, P. J.; Patel, D. S.; Wu, E. L.; Qi, Y.; Yeom, M. S.; Sousa, M. C.; Fleming, K. G.; Im, W. BamA POTRA Domain Interacts with a Native Lipid Membrane Surface. Biophys. J. 2016, 110 (12), 2698-2709.

(93) Patel, D. S.; Park, S.; Wu, E. L.; Yeom, M. S.; Widmalm, G.; Klauda, J. B.; Im, W. Influence of Ganglioside GM1 Concentration on Lipid Clustering and Membrane Properties and Curvature. Biophys. J. 2016, 111 (9), 1987-1999.

(94) Lee, J.; Patel, D. S.; Kucharska, I.; Tamm, L. K.; Im, W. Refinement of OprH-LPS Interactions by Molecular Simulations. Biophys. J. 2017, 112 (2), 346-355.

(95) Patel, D. S.; Qi, Y.; Im, W. Modeling and Simulation of Bacterial Outer Membranes and Interactions with Membrane Proteins. Curr. Opin. Struct. Biol. 2017, 43, 131-140.

(96) Blasco, P.; Patel, D. S.; Engström, O.; Im, W.; Widmalm, G. Conformational Dynamics of the Lipopolysaccharide from Escherichia Coli O91 Revealed by Nuclear Magnetic Resonance Spectroscopy and Molecular Simulations. Biochemistry 2017, 56 (29), 38263839.

(97) Matthias, K. A.; Strader, M. B.; Nawar, H. F.; Gao, Y. S.; Lee, J.; Patel, D. S.; Im, W.; Bash, M. C. Heterogeneity in Non-Epitope Loop Sequence and Outer Membrane Protein Complexes Alters Antibody Binding to the Major Porin Protein PorB in Serogroup B Neisseria Meningitidis. Mol. Microbiol. 2017, 105 (6), 934-953.

(98) Feigman, M. S.; Kim, S.; Pidgeon, S. E.; Yu, Y.; Ongwae, G. M.; Patel, D. S.; Regen, S.; Im, W.; Pires, M. M. Synthetic Immunotherapeutics against Gram-Negative Pathogens. Cell Chem. Biol. 2018.

(99) Lee, J.; Pothula, K. R.; Kleinekathöfer, U.; Im, W. Simulation Study of Occk5 Functional Properties in Pseudomonas Aeruginosa Outer Membranes. J. Phys. Chem. B 2018, 122 (34), 8185-8192. 